\title{
A Detailed 3D Model of the Rabbit Right Atrium Including the Sinoatrial Node, Atrioventricular Node, Surrounding Blood Vessels and Valves
}

\author{
$\mathrm{J} \mathrm{Li}^{1}$, JE Schneider ${ }^{2}, \mathrm{M}$ Yamamoto ${ }^{1}$, ID Greener ${ }^{3}$, \\ H Dobrzynski ${ }^{1}$, K Clarke ${ }^{2}$, MR Boyett ${ }^{1}$ \\ ${ }^{1}$ Division of Cardiovascular and Endocrine Sciences, University of Manchester, UK \\ ${ }^{2}$ Department of Cardiovascular Medicine, John Radcliffe Hospital, UK \\ ${ }^{3}$ School of Biomedical Sciences, University of Leeds, UK
}

\begin{abstract}
We used multiple techniques to generate a threedimensional anatomical model of the rabbit right atrium with the sinoatrial node (SAN) and atrioventricular node $(A V N)$. The model includes the right atrium, SAN, AVN, part of right ventricle, aorta with aortic valve, superior vena cava, inferior vena cava, coronary sinus, tricuspid valve, part of mitral valve, fossa ovalis and central fibrous body. The tendon of Todaro and right and left sinoatrial ring bundles were highlighted as landmarks.
\end{abstract}

\section{Introduction}

Accurate simulation of the generation and propagation of cardiac electrical activity requires detailed electrophysiological and anatomical models. We previously generated geometric models of the sinoatrial node (SAN) and atrioventricular node (AVN) based on histology and immunolabelling of serial sections cut from SAN and AVN preparations (Dobrzynski et al., 2005; Li et al., 2004). The purpose of this study was to construct a three dimensional (3D) anatomical model of the rabbit right atrium with multiple objects, which include the SAN, AVN, surrounding blood vessels and valves. The model can be used to help understand the detailed anatomy of the right atrium (especially the conduction system), and, in combination with the electrophysiological models, to simulate the propagation of electrical activity within the right atrium.

\section{Methods}

$\sim 1500$ images of the rabbit heart (the two atria and part of the two ventricles) were obtained by high-resolution magnetic resonance imaging (MRI) at an $11.7 \mathrm{~T}$ Bruker MR system (Bruker Medical, Ettlingen, Germany). The shape of right atrium was maintained in the natural state by the remaining two ventricles and the left atrium. The voxel size after reconstruction was $26.4 \mu \mathrm{m} \times 26.4 \mu \mathrm{m} \times$
$24.4 \mu \mathrm{m}$.
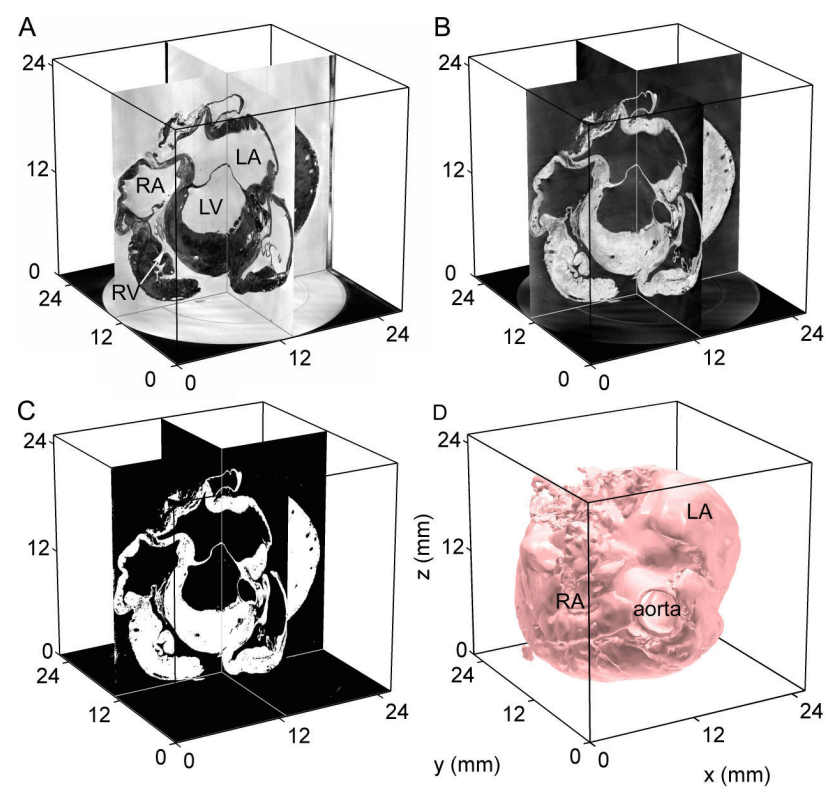

Figure 1. A, isotropic $3 \mathrm{D}$ array through three compact orthogonal slices. RA, right atrium; LA, left atrium; RV, right ventricle; LV, left ventricle. $\mathrm{B}$, corrected and complemented array. C, converted binary array (3D array model). $\mathrm{D}$, the first 3D model (isosurfaces).

MATLAB (version 7; The MathWorks, Inc., Matick, MA, USA) was used to analyse the data and reconstruct the 3D multiple objects rabbit right atrium model. The $\sim 1500$ two-dimensional (2D) images were imported into the workspace of MATLAB and stacked to produce a 3D array with anisotropic elements. The anisotropic 3D array was converted to an isotropic $\left(30 \mu \mathrm{m}^{3}\right) 3 \mathrm{D}$ array using cubic interpolation. Figure 1A shows the isotropic array through three compact orthogonal slices. Four chambers (right atrium, left atrium, right ventricle and left ventricle) can be recognised. A cylindrical shape can be seen outside of the structure, because the specimen was scanned within a cylindrical probe. The array had to be 
corrected by erasing the unwanted cylindrical boundary. The complement of the array was obtained (Figure 1B) to extract the structure (the voxel values of the structure were higher than those of the background). The complemented array was converted to a binary array based on a global threshold (Figure 1C). For visualization, isosurfaces were then used to display the overall structure of the 3D model (Figure 1D). This model includes the two atria and part of the two ventricles without segmentation. The aorta can be recognised and this can be used as a reference structure for detecting the anatomical orientation.
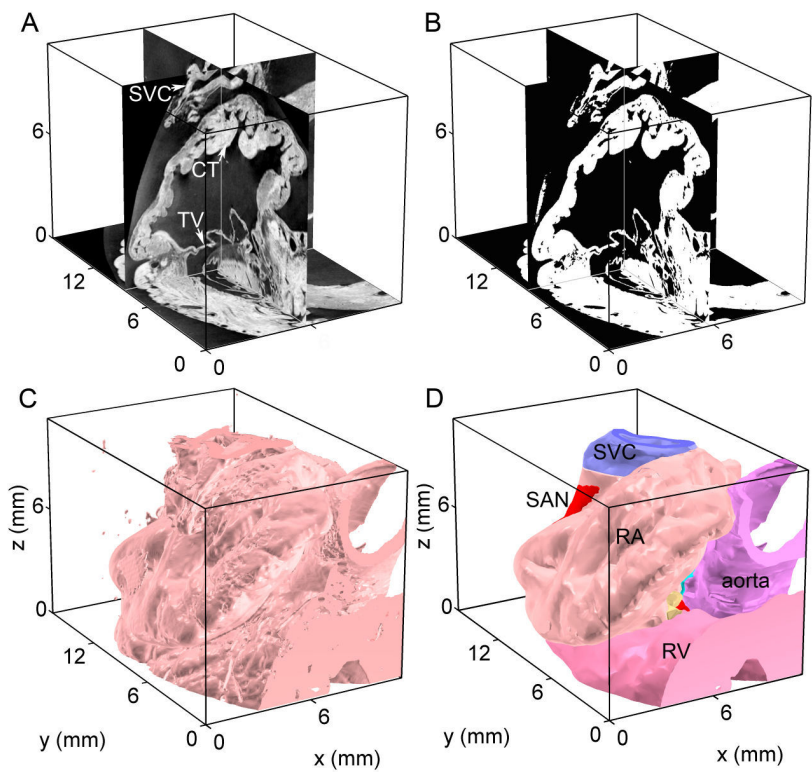

Figure 2. Model with correct anatomical orientation. Only the right atrium is shown - the left atrium and ventricles have been 'cut' off. A, the right atrium array through three compact orthogonal slices. SVC, superior vena cava; CT, crista terminalis; TV, tricuspid valve. B, binary array. C, 3D isosurfaces model without segmentation. D, 3D isosurfaces model with segmentation.

After analysis of the first 3D model, the 3D array was rotated to the correct anatomical orientation ( $\mathrm{x}$, lateralmedial; y, anterior-posterior; $\mathrm{z}$, inferior-superior). Our aim is to construct a 3D right atrium model. Hence, the left atrium, left ventricle and part of right ventricle were 'cut' off. Figure 2 shows the model of the right atrium with the correct anatomical orientation. Figure 2A shows the rotated and complemented right atrium array through three compact orthogonal slices. The superior vena cava (SVC), crista terminalis (CT) and tricuspid valve (TV) can be recognised. Figure $2 \mathrm{~B}$ shows the converted binary array. Figure 2C shows the 3D right atrium model (isosurfaces shown) without any segmentation. Figure 2D shows the 3D right atrium model after segmentation.

Segmentation was carried out semi-manually based on the binary array (Figure 2B) by analysing the MRI data from three directions (along $\mathrm{x}, \mathrm{y}$ and $\mathrm{z}$ axes). Figure 3 shows the procedure of the segmentation used in this study - segmentation of the aorta with the aortic valve is shown as an example. First, we investigated the 3D MRI array from three directions to choose the best orientation for segmentation (along $\mathrm{x}, \mathrm{y}$ or $\mathrm{z}$ axes). For segmenting the aorta with the aortic valve, the best orientation was along the $\mathrm{z}$ axis (x-y plane; Figure 3A). Second, the object was extracted from the structure every 5 10 slices (Figure 3B). In Figure 3B, the upper image is an MRI image and the lower image is the segmented object image. Then interpolation was carried out to obtain the 3D object. Figure 3C shows the object from another direction ( $\mathrm{X}-\mathrm{Z}$ plane). In Figure 3C, the upper image is an MRI image. The middle and lower images are segmented object images before and after interpolation. Finally, the object was combined with other objects to be a multiobject model image (Figure 3D). Thirteen objects were segmented in this study.

A. Choose best orientation

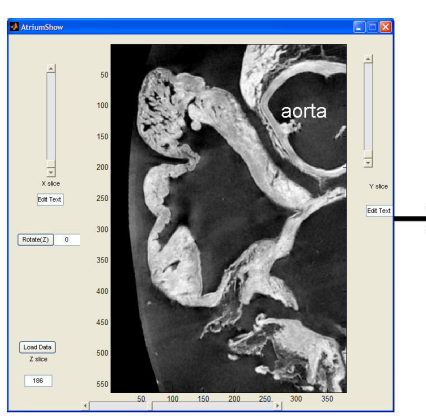

D. Combine with other objects

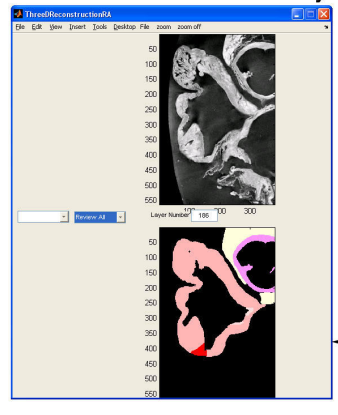

Figure 3. Typical segmentation procedure used in this study (segmentation of the aorta with the aortic valve is shown as an example.

The right atrium, a small part of the right ventricle, the aorta with the aortic valve, the coronary sinus, the tricuspid valve, part of the mitral valve and the fossa ovalis are easy to recognise in the MRI images and were segmented as just described. 


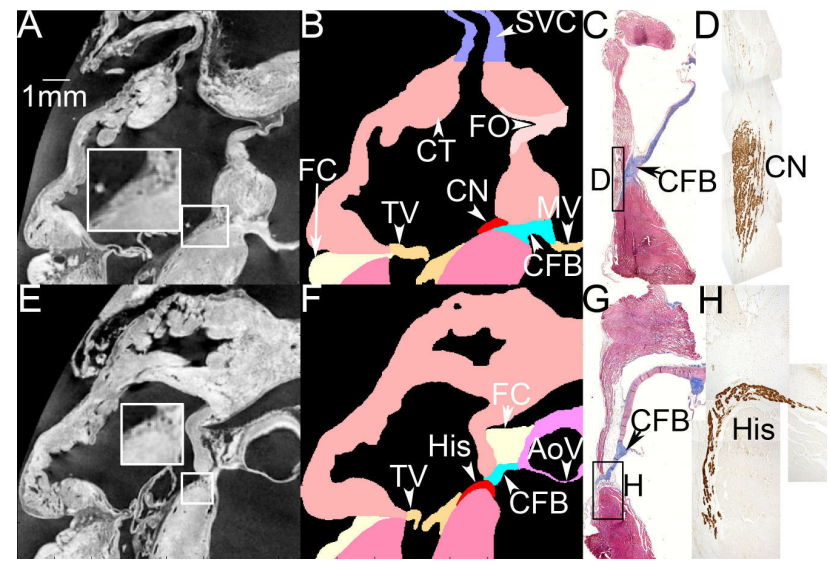

Figure 4. A, MRI image of the compact AVN. The AVN region is enlarged in the inset (from the boxed area shown). B, model section after segmentation based on A. FO, fossa ovalis; $\mathrm{CN}$, compact $\mathrm{AVN}$; $\mathrm{CFB}$, central fibrous body; MV, mitral valve; FC, outer fatty and connective tissue. $\mathrm{C}$, section through the compact part of the AVN stained with Masson's trichrome. D, section through the AVN region in C labelled for neurofilament. The neurofilament-positive cells are AVN cells. E, MRI image including the His bundle. The His bundle region is enlarged in the inset (from the boxed area shown). F, model section after segmentation based on E. His, His bundle; AoV, aortic valve. G, section through the His bundle stained with Masson's trichrome. $\mathrm{H}$, section through the His bundle region in $G$ labelled for neurofilament. The neurofilament-positive cells comprise the His bundle.

The SAN and AVN were detected by comparing the MRI images with the histology of the two regions and our two previous 3D models ( $\mathrm{Li}$ et al., 2004). It was found that the AVN could be distinguished in the MRI images after comparing the MRI images with histological sections of the region. Figure 4 shows how we detected the AVN by comparing the MRI image with a histological section and a neurofilament imunolabelled section. Figure 4A-D shows the images through the compact part of the AVN. Figure 4E-H shows the images through the His bundle. A similar pattern can be distinguished in Figure 4A (MRI image) and Figure 4C (section stained with Masson's trichrome), and Figure 4E (MRI image) and Figure 4G (Masson's trichrome). The central fibrous body can be distinguished in the MRI images by comparing $\mathrm{A}$ with $\mathrm{C}$ and $\mathrm{E}$ with $\mathrm{G}$ in Figure 4 . Figure 4D, H shows sections labelled for neurofilament. The neurofilament-positive cells are AVN cells. The AVN can be distinguished in the MRI image by comparing panel A with panel $\mathrm{C}$ and panel $\mathrm{E}$ with panel $\mathrm{G}$ in Figure 4. From the enlarged images within Figure $4 \mathrm{~A}, \mathrm{E}$, the AVN could be extracted from the surrounding atrial muscle. Figure $4 \mathrm{~B}, \mathrm{~F}$ shows sections from the segmented multi-objects model based on Figure 4A, E.
Some artificial correction was carried out when the SVC and the tricuspid valve were segmented, because the SVC was bent and the tricuspid valve was flipped as seen in Figure 4A, E.

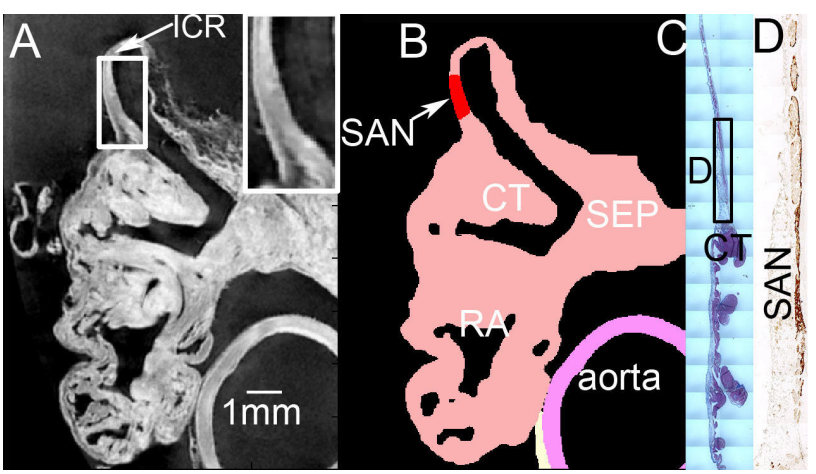

Figure 5. A, MRI image including the intercaval region. ICR, intercaval region. The SAN region is enlarged in the inset (from the boxed area shown). B, the model section after segmentation based on A. SEP, interatrial septum. $\mathrm{C}$, section through the SAN stained with Masson's trichrome. D, SAN region in $\mathrm{C}$ labelled for neurofilament. The neurofilament-positive cells are SAN cells.

For SAN segmentation, we compared MRI images (Figure 5A) with histological sections (Figure 5C) with similar patterns. From the enlarged image within Figure $5 \mathrm{~A}$, it is impossible to extract the SAN from the surrounding atrial muscle as we did for the AVN. To define the central region of the SAN, we measured the distance between the crista terminalis and the SAN centre and the length of SAN centre in histological sections (Figure 5C, D). The result is shown in Figure 5B. The SAN periphery has not been included in this model due to its complexity (Li et al., 2004).

The location of the tendon of Todaro and the right and left sinoatrial ring bundles within the 3D array were detected by analysing the MRI images and histological sections. However, this information has not been included in the 3D array.

\section{Results}

A 3D right atrium array model including thirteen objects was constructed. The objects are the right atrium, the SAN, the AVN, part of the right ventricle, the aorta with the aortic valve, the superior vena cava (SVC), the inferior vena cava (IVC), the coronary sinus, the tricuspid valve, part of the mitral valve, the fossa ovalis, the central fibrous body and outer fatty and connective tissue.

For visualization, the 3D model was displayed by isosurfaces for visualisation. Twelve out of the thirteen objects were displayed. The outer fatty and connective tissue was not displayed in the 3D isosurfaces model. The 
tendon of Todaro and the right and left sinoatrial ring bundles were highlighted using spline lines.

Figure 6 shows the 3D isosurface model from different views. Figure $6 \mathrm{~A}$ shows a laterior-posterior view of the model. The entire outer shape of the right atrium can be seen from this view. The right atrial appendage can be recognised easily. The SAN is located at the posterior side, in the intercaval region. The SVC and the IVC are approximately orthogonal to each other. Figure 6B shows a posterior view of the model. The coronary sinus runs under the IVC, approximately orthogonal with the IVC. The orientation of the SVC is, approximately along the $\mathrm{z}$ axis (inferior-superior), the orientation of the IVC is, approximately along the $y$ axis (anterior-posterior) and the orientation of the coronary sinus is, approximately along the $\mathrm{x}$ axis (lateral-medial). Figure $6 \mathrm{C}$ shows a part of the model from an anterior-medial view. The SAN centre is located within the intercaval region and is surrounded by the right and left sinoatrial ring bundles. Figure 6D shows a part of the model from an anteriorlateral view. The His bundle can be seen. The compact part of the AVN lies at the end of the tendon of Todaro. The fossa ovalis can also be seen in this view.

A

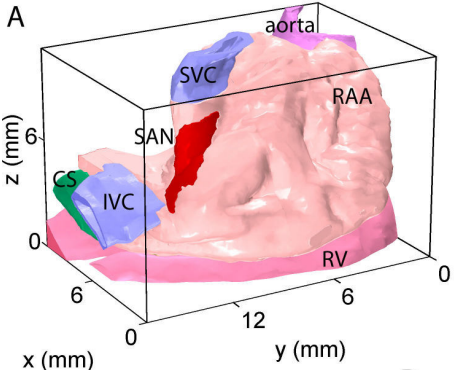

B
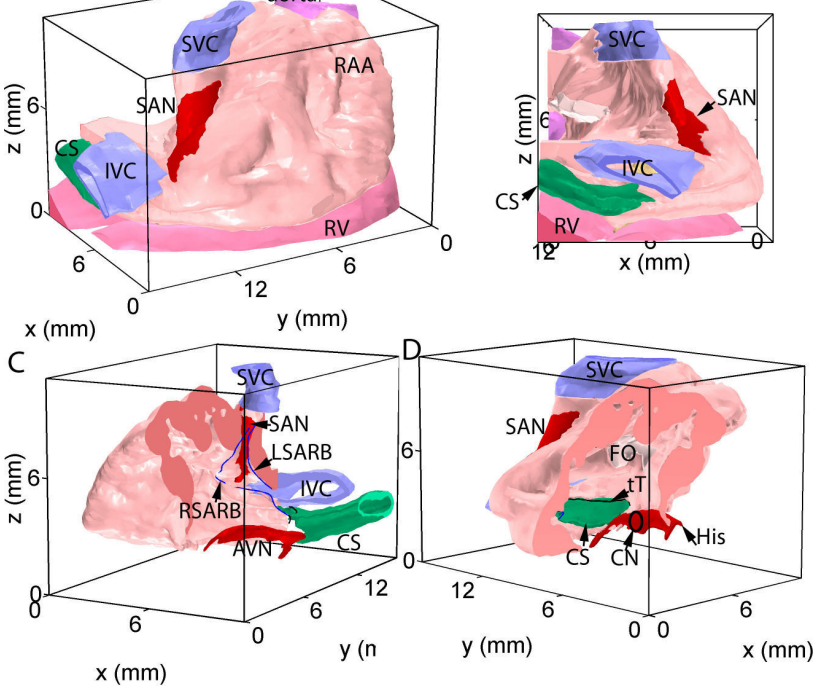

Figure 6. A, lateral-posterior view of the 3D multiobjects isosurface right atrium model. RAA, right atrial appendage. B, posterior view of the model. $\mathrm{C}$, anteriormedial view of part of the model showing inside the structure. RSARB, right sinoatrial ring bundle; LSARB, left sinoatrial ring bundle. $\mathrm{D}$ anterior-lateral view of part of the model. $\mathrm{tT}$, tendon of Todaro.

\section{Discussion}

We developed the 3D model of the right atrium with the SAN and AVN based on high resolution MRI data of the rabbit heart combined with the analysis of histological and immunolabelled serial sections through the SAN and AVN. It was a delight to discover that the AVN could be distinguished in the MRI data. Unfortunately, the SAN could not be so easily distinguished in the MRI data.

Initially, we proposed to segment the SAN by transforming the previous SAN model (Dobrzynski et al., 2005 ) onto the $3 \mathrm{D}$ right atrium model using reference points (the right and left sinoatrial ring bundles etc.). In this case, the SAN periphery should have been obtained. However, this approach was unsuccessful due to the complexity and variability of the muscle bundles within the right atrium.

The reason for developing the 3D model of the right atrium with the SAN and AVN (conduction system) is to simulate the generation and propagation of electrical activity through the tissue. Cell orientation is important for propagation. Unfortunately, it is impossible to detect cell orientation from the MRI data. It is possible to detect muscle bundles within the right atrium free wall from the MRI data. It is reasonable to hypothesise that the cells run longitudinally along the muscle bundles. However, it is impossible to infer cell orientation within the intercaval region and interatrial septum from the MRI data in the same way. Further work, therefore, is still required.

\section{Acknowledgements}

The work is supported by the British Heart Foundation.

\section{References}

[1] Li J, Dobrzynski H, Greener ID, Nikolski VP, Yamamoto M, Billeter R, Efimov IR \& Boyett MR. Development of 3D anatomically-detailed mathematical models of the sinoatrial and atrioventricular nodes. Computers in Cardiology. 2004; 31:89-92.

[2] Dobrzynski H, Li J, Tellez J, Greener ID, Nikolski VP, Wright SE, Parson SH, Jones SA, Lancaster MK, Yamamoto M, Honjo H, Takagishi Y, Kodama I, Efimov IR, Billeter R \& Boyett MR. Computer three-dimensional reconstruction of the sinoatrial node. Circulation. 2005; 111:846-854.

Address for correspondence

Professor Mark .R. Boyett

Division of Cardiovascular and Endocrine Sciences

University of Manchester

Manchester Incubator Building

48 Grafton Street

Manchester

M13 9XX, UK

mark.boyett@manchester.ac.uk 